\title{
Pattern of Intracranial and Extracranial Projections of Trigeminal Ganglion Cells
}

\author{
Timothy P. O'Connor and Derek van der Kooy \\ Neurobiology Research Group, Department of Anatomy, University of Toronto, Toronto, Ontario, \\ Mi5S 1A8, Canada
}

\begin{abstract}
The trigeminal sensory innervation of the major cerebral vessels is thought to carry the nociceptive information during a migraine headache, and this pain is usually referred to the forehead area. Using retrograde tracing techniques, we have described the distribution of sensory trigeminal cells that innervate the middle cerebral artery (MCA) and the forehead. Nearestneighbor analysis of the ophthalmic division of the trigeminal ganglion revealed that cells innervating the forehead tend to be clumped around individual cells that innervate the MCA. An average of less than 1 cell per animal was found to project divergent collaterals to both areas. The close association of ganglion cell bodies innervating the cerebral vasculature and those innervating forehead areas may underlie the convergence of their central processes onto common brain-stem trigeminal nucleus cells, and thus the referral of headache pain.

In contrast to the lack of ganglion cells with axonal collaterals to the cerebral vasculature and forehead, a significant population of cells that innvervate the MCA also have collateral projections to other cerebral arterial branches (branches of the middle meningeal artery), as well as the surrounding dura. Thus, the innervation targets of individual trigeminal cells are very widespread intracranially (including arteries and dura), but separate cells in the ophthalmic division innervate extracranial targets.
\end{abstract}

The presence of nerves in the dura, major cerebral arteries, extradural artcrics, venous sinuses, and pial arteries has long been documented (Gulland, 1898; Huber, 1899; Penfield, 1932; Penfield and McNaughton, 1940). Modern axonal transport techniques have only recently documented projections specifically from the trigeminal ganglion to the cerebral arteries arising from the circle of Willis and to the dura (Mayberg et al., 1981; Steiger et al., 1982). These projections are thought to carry the nociceptive information during a migraine (Moskowitz, 1984) or cluster headache (Hardebo, 1984). This pain is usually referred to a peripheral receptive field. Penfield and McNaughton (1940), Ray and Wolff (1940), and Wirth and Van Buren (1971) demonstrated the referral of pain from arterial and dural stimulation in man. They showed wide areas of dura and dural sinus stimulation gave rise to referred pain in the eye, forehead, temple, and neck region. Stimulation of the proximal middle cerebral artery (MCA) also produces pain ipsilaterally in the forehead or behind the eye (Fay, 1932).

The location where nociceptive and cutaneous information converge, resulting in the phenomenon of referred pain, is still

Received July 23, 1985; revised Dec. 13, 1985; accepted Jan. 24, 1985.

This work was supported by the Medical Research Council of Canada, the Onlario Heart and Stroke Foundation, and a Canadian Heart Foundation Research Traineeship (T.O.)

Correspondence should be addressed to Tim O'Connor at the above address. Copyright (C) 1986 Society for Neuroscience $0270-6474 / 86 / 082200-08 \$ 02.00 / 0$ under debate. Attention has focused primarily on secondary and tertiary sensory neurons in the dorsal horn of the spinal cord and trigeminal sensory complex (Hu et al., 1981; Milne et al., 1983), but more peripheral sites of convergence are also under consideration (Pierau et al., 1984; Taylor and Pierau, 1982). With respect to headache, Mayberg et al. (1981) have suggested the existence of bifurcating axons from single trigeminal ganglion cells to both the middle cerebral artery (MCA) and the forehead region as a putative mechanism for the referred pain. Little evidence for this anatomical arrangement was found in either monkey (Borges and Moskowitz, 1983) or rat (O'Connor and van der Kooy, 1983). However, it was anecdotally observed that cells innervating the MCA frequently had a forehead cell close by (Borges and Moskowitz, 1983; O'Connor and van der Kooy, 1983). In the present paper a detailed analysis is made of the close relationship between the populations of trigeminal ganglion cells innervating the forchead and MCA. Furthermore, we report the existence of single trigeminal ganglion cells with divergent axon collaterals to various intracranial targets, including cerebral arteries, subdural arteries, and the dura itself.

\section{Materials and Methods}

Male Charles River rats were anesthetized with intraperitoneal sodium pentobarbital (Somnotol; $65 \mathrm{mg} / \mathrm{kg}$ ). The fluorescent retrograde axonal tracers employed were 5\% True Blue (TB) and 2\% Diamidimo Yellow (DY) (both obtained from Dr. Illing, FRG, and dissolved in distilled water). The specific sites of tracer application are listed after a description of the general methods. After various survival times, animals were deeply anesthetized and transcardially perfused with $100 \mathrm{ml}$ saline $(0.9 \%)$ followed by $300-400 \mathrm{ml} 10 \%$ formalin. The brain and trigeminal ganglia were removed and stored in $20 \%$ sucrose overnight; they were then sectioned at $32 \mu \mathrm{m}$ on a cryostat at $-14^{\circ} \mathrm{C}$. All sections from the ganglia ipsilateral to the artery application were saved, and every second or third section was saved from the contralateral ganglia. Brains were also sectioned in order to examine any spread of the tracers. Sections were examined under a Leitz epifluorescence microscope with excitation at $340-380 \mathrm{~nm}$.

\section{Retrograde labeling from the MCA versus forehead}

The MCA was isolated from a parietotemporal approach. The dura was cut and reflected away from the artery. The pia arachnoid on both sides of the artery was cut, and a small triangular piece of parafilm (approximately $1 \times 2 \mathrm{~mm}$ ) was pulled through underneath the artery. Viscous $5 \%$ TB (30-60 nl) was applied to the artery, and the TB was allowed to dry and adhere to the outer adventitia. In some cases, the parafilm was then removed; in others, it was left in place underneath the artery. The artery was then covered by 2 layers of gel foam, and another outermost layer of parafilm. The cut muscle and skin were sutured and the animal allowed to recover. Some 3 to $4 \mathrm{~d}$ later, the animals were reanesthetized, and the frontal branch of the ophthalmic nerve was dissected out just distal to the supraorbital foramen and cut. The transected end was placed in a small plastic tube containing $2 \%$ DY. The tube was fixed to the skull and covered by a layer of gel foam. The rats were sacrificed $24 \mathrm{hr}$ later.

To obtain nearest-neighbor measurements betwecn retrogradcly la- 
beled cells in single sections through the trigeminal ganglion, pictures were taken of all the TB-labeled cells (and surrounding areas) with Kodak Ektachrome color film. Photographed areas were noted on the microscope axis coordinates. The sections were stained with $0.1 \%$ cresyl violet and were then dehydrated and defatted in increasing concentrations of alcohol and xylene. After cresyl violet staining, the previously photographed areas were rephotographed through the same microscope under bright-field illumination with Kodak black-and-white Pan-X film. On each roll of film, a picture was also taken of a $1 \mathrm{~mm}$ calibrating line at the same magnification. A collage of the pictured areas was made using the black-and-white pictures, and corresponding cells that were retrogradely labeled from the artery and forehead injections were marked. Nearest-neighbor analysis was used to determine if cells projecting to the artery and forehead were clumped or randomly distributed (Clark and Evans, 1954, 1979). Measurements between retrogradely labeled cells were made directly from the black-and-white collages with an Osborne 1 microcomputer and Summagraphic digitized Bit Pad (Lea, 1983). Distance measurements between cells were made from the plasma membrane of the cell body in question to the plasma membrane of its corresponding nearest-neighbor. Three different nearest-neighbor measurements were made: (1) each artery projecting cell to its nearest artery projecting neighbor, (2) each forehead projecting cell to its nearest forehead projecting neighbor, and (3) each artery projecting cell to its nearest forehead projecting neighbor.

Using the same Nissl pictures, cell areas from the 2 populations were measured. Only those cells with a nucleus were measured. Cell diameters were calculated from these data.

\section{Retrograde labeling from the dura}

To determine whether there were projections from the trigeminal ganglia to the dura, TB soaked gel foam was applied to the dura in areas where there were no major arteries or veins (Fig. $4 A$ ). A small cut was made in the dura at the site of application to ensure exposure of the tracer to any nerves running in the dura, and the TB was covered by 2 layers of gel foam and a strip of parafilm as described above. The animals were allowed to survive 4-5 d and were then sacrificed and perfused as described earlier. Sections of the brain and ganglia were taken and examined under the epifluorescence microscope.

\section{Retrograde labeling from $M C A$ versus dura or middle meningeal artery (MMA)}

Four procedures were performed to determine the extent of the projections from the trigeminal ganglion to various intracranial arteries and dural targets. Figure 5 shows the various application sites of the 2 tracers. First, the MCA was isolated, and TB was applied exactly as described above to 3 rats. At the same time, the anterior branch of the MMA (aMM) was isolated in a similar manner, and $30-60 \mathrm{nl}$ of DY was applied to this artery (Fig. 5, no. 4). The arteries were covered and the wound closed as described above. To allow transport for both tracers, the animals were allowed to survive for $\mathbf{4} \mathrm{d}$ before they were sacrificed and the tissue processed as above.

Second, the MCA was isolated and TB was applied in 2 rats. At the same time, the dura was cut between the anterior and middle branches of the MMA and $2 \%$ DY was applied to the cut edge of the dura (Fig. 5 , no. 2). No large arteries or veins were present at the site of DY application.

Third, TB was applied to the MCA, and DY was applied to the middle branch of the MMA (mMM) in two rats (Fig. 5, no. 5).

Fourth, TB was applied to the MCA, and DY was applied to various dural sites in 3 rats. Sites of DY application were either anteriodorsal (Fig. 5, no. 1) or just posterior to the MCA (Fig. 5, no. 3).

Additional experiments were performed to ensure that trigeminal labeling was not due to spread to other trigeminal innervated targets and to determine the course of the trigeminal afferents to the arteries. In 2 animals the MCA was isolated and TB applied as described above. Immediately following the TB application, the artery was cauterized proximally. The application was covered and the animal sutured as above. In another experiment, the aMM artery was isolated in 2 animals at a site similar to that shown in Figure 5, no. 4. In one animal, the artery was cauterized proximal to the TB application, and in the second, the artery was cauterized proximal and distal to the TB. Cauterizations were performed immediately following the TB applications. After $5 \mathrm{~d}$ the animals were sacrificed and processed as above. If the nerves run in a proximal to distal direction, a proximal lesion alone should prevent
A

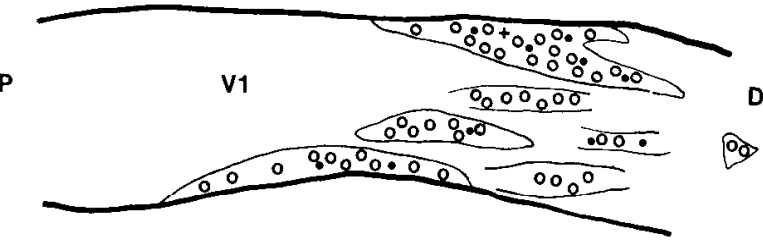

B
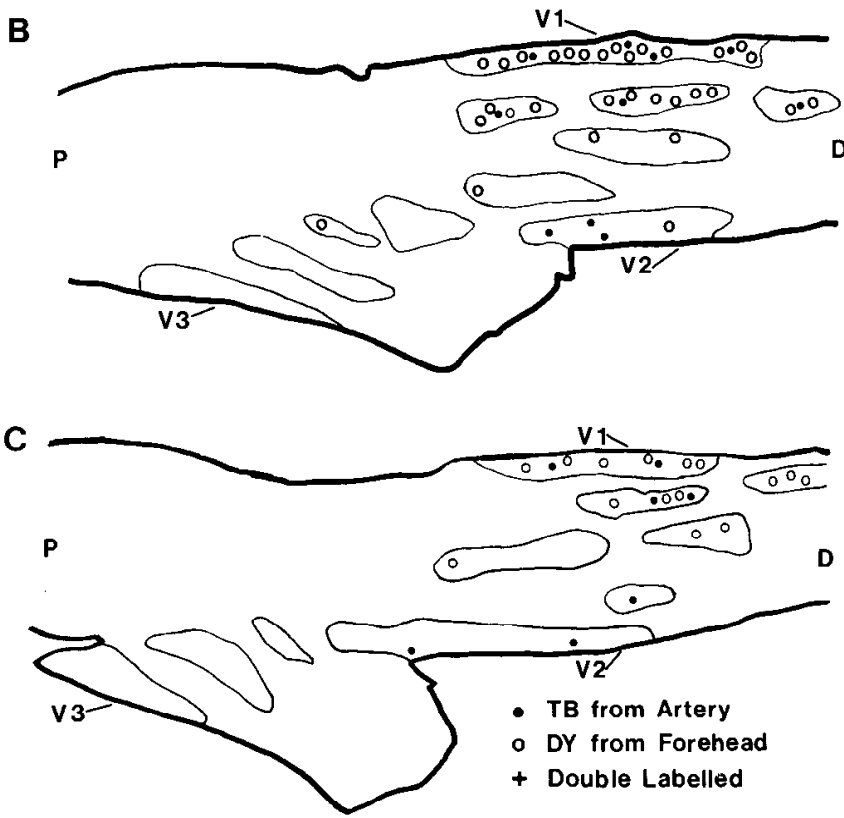

Figure 1. Distribution of MCA (TB, $\bullet$ ), forehead (DY, O), and dual (TB and DY, + ) projecting cells in the trigeminal ganglion of the rat. These are horizontal sections from the right (ipsilateral) ganglion. Cells schematized are representative of the distributions from several sections. $A$, Dorsal-most section (which includes only the ophthalmic division) has both artery- and forehead-projecting cells throughout the medial to lateral extent of the ganglion. An average of less than 1 cell per rat was double-labeled $(+)$. $B$, In intermediate sections, arteryinnervating cells are found in both the ophthalmic ( $V I)$ and maxillary (V2) divisions of the ganglia. $C$. Ventral sections contain fewer cells but with a distribution similar to that seen in the intermediate sections. The average number of TB cells per animal was $45 \pm 8.6(n=12)$. Abbreviations: $P$, proximal; $D$, distal; $V 1$, ophthalmic branch; $V 2$, maxillary branch; $V 3$, mandibular branch.

all retrograde labeling. If, however, there is some nerve exchange where the aMM crosses over the MCA artery, then some of these nerves may run in a distal to proximal direction. In this latter case, only combined proximal and distal lesions would be expected to prevent all retrograde labeling.

\section{Results}

\section{Innervation of $M C A$ versus forehead}

Differences were seen in the distributions of trigeminal ganglion cells projecting to the MCA versus those projecting to the forehead (Fig. 1). All of the numerous cells innervating the forehead region were found in the ophthalmic branch of the ganglia. In the most dorsal part of the ganglion, labeled cells were observed over its entire medial to lateral extent, with a majority of the cells in the anteriomedial area of the ganglion. More ventrally, cells were found only in this anteriomedial area of the ganglion. These observations support the parcellation of the rat trigeminal ganglion as observed by Mazza and Dixon (1972) and Gregg and Dixon (1973). They determined that the ophthalmic branch was located anteriomedially, the mandibular branch posteriolaterally, and the maxillary branch between these 2 divisions. 


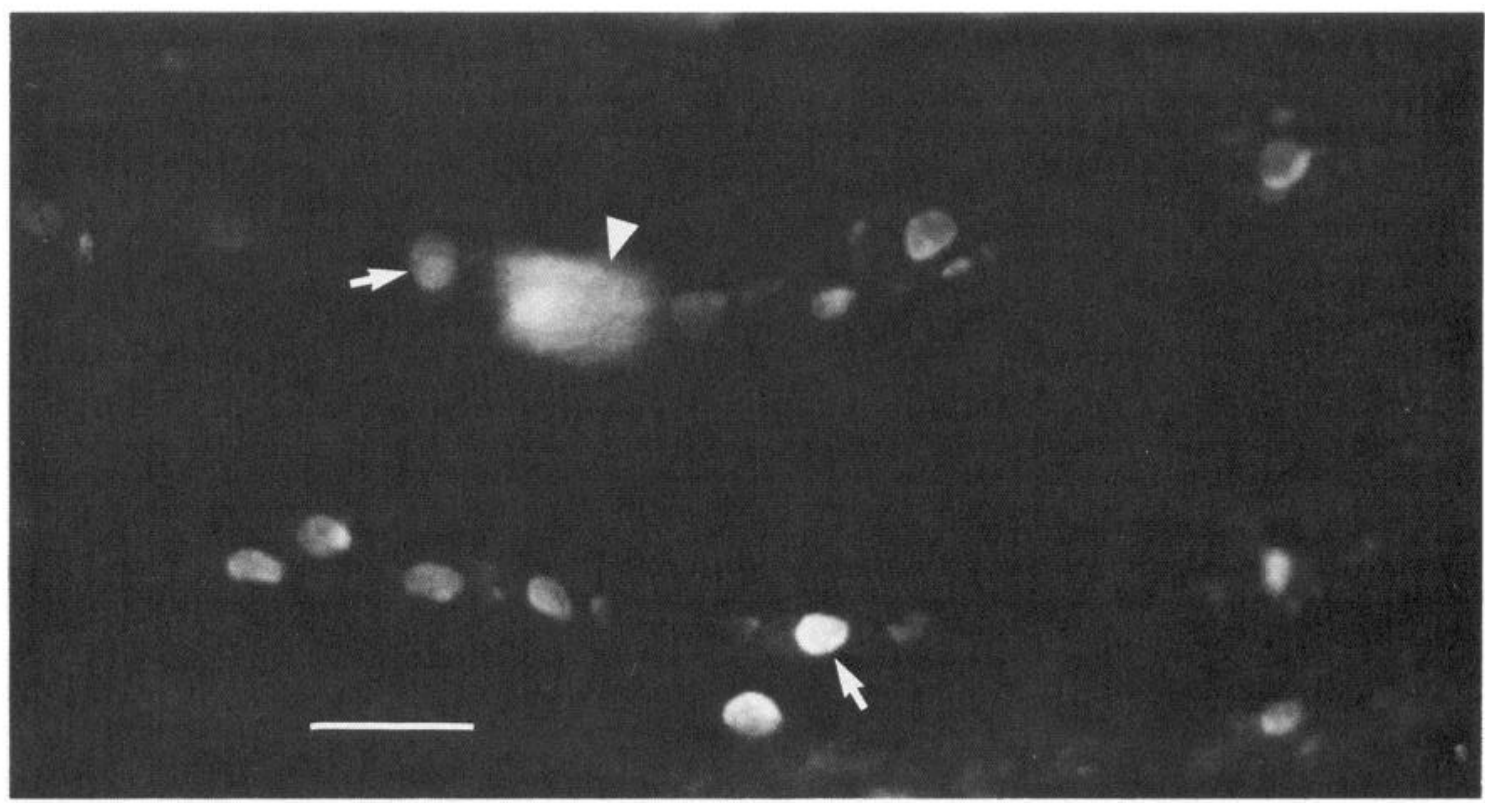

Figure 2. Photomicrograph of the retrograde labeling of trigeminal cells after TB was applied to the MCA and DY to the frontal nerve. Arteryprojecting cells usually have a forehead-projecting cell nearby. Labeled cells can be distinguished by 2 criteria, the color and the intracellular location of the tracer. TB is seen as a fine blue fluorescence located diffusely throughout the cytoplasm of the cell (arrowhead), whereas the DY is a bright, white-yellow fluorescence of the nucleus of the cells (arrows). In some cases, DY appeared to have leaked out of the cell, and nearby glial cells were labeled. To limit this, we used survival times that resulted in optimal neuronal with minimal glial labeling. An average of less than 1 cell per rat was double-labeled in these cases. Bar, $40 \mu \mathrm{m}$.

An average of 45 cells per rat (SEM $= \pm 8.6, n=12$ rats) in the ipsilateral ganglion was found to project to the MCA. Of these artery projecting cells $60 \pm 5 \%$ were found in the ophthalmic branch among the cells innervating the forehead and $38 \pm 5 \%$ were found in the maxillary branch. A few cells retrogradely labeled from the artery $(2 \pm 1.0)$ were found in the mandibular branch of the ganglia. Very few cells (an average of less than 1 per animal, i.e., approximately $1 \%$ of the cells labeled from the MCA), were double-labeled, indicating that very few ganglion cells projected to both the MCA and the forehead.
Figure 3. Diameters of trigeminal cells that project to the forehead $(-)$ and the MCA (-.--) in the rat. The mean diameter of forehead cells was $24 \pm 0.8 \mu \mathrm{m}$ and that of the arteryprojecting cells, $28 \pm 0.9 \mu \mathrm{m}$. The artery-innervating cells tended to have a greater proportion of large cells than did the forehead population.

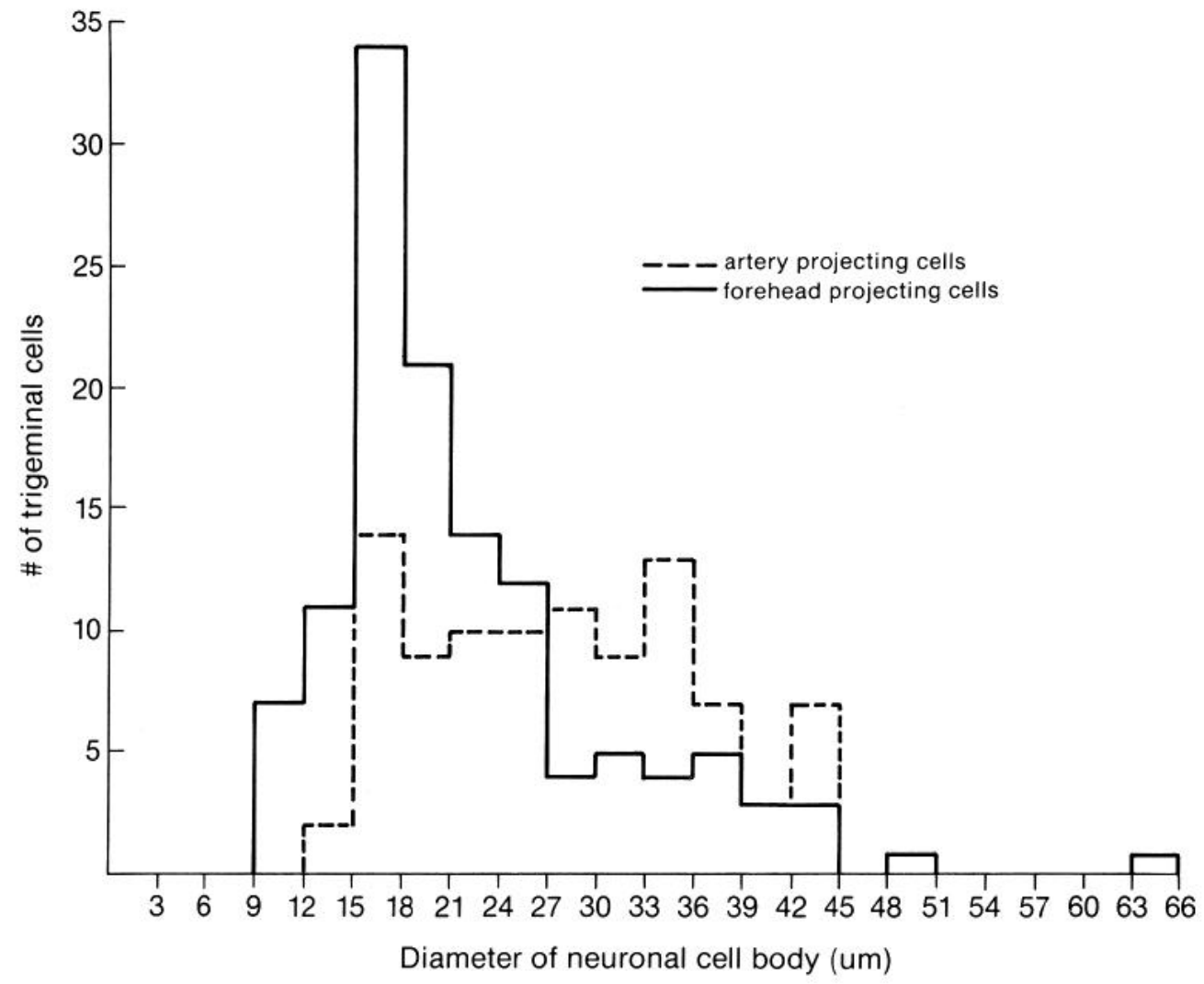


A
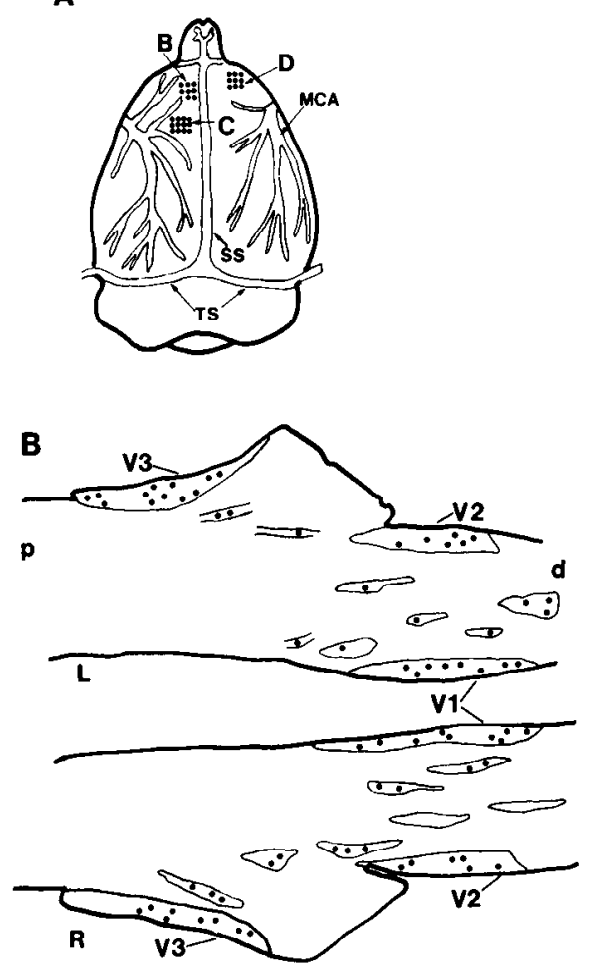
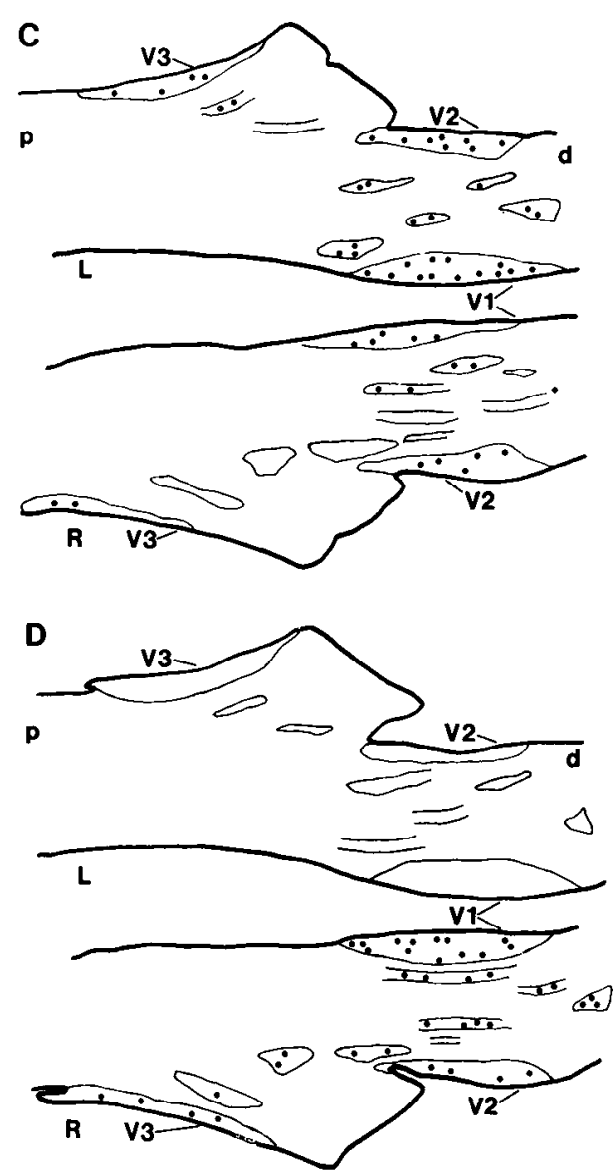

Figure 4. Representation of the dural innervation of the trigeminal ganglia after application of TB $(A, \bullet)$ to different dural sites $(B-D)$. As the application of TB was moved medially, a number of retrogradely labeled cells appeared in the contralateral ganglia. Abbreviations: $P$, proximal; $D$, distal; $V 1$, ophthalmic branch; V2, maxillary branch; V3, mandibular branch; $L$, left; $R$, right; $T S$, transverse sinus; $S S$, sagittal sinus.
Nearest-neighbor measurements were made from pictures of 9 sections from one ganglion and 12 sections from another ganglion. Only the populations of cells in the ophthalmic branch were included in the analysis. The nearest-neighbor measurement uses a ratio $R=\bar{r}_{\mathrm{ob}} / r_{\mathrm{e}}$, where $P_{\mathrm{ob}}$ is the mean nearestneighbor observed, while $\bar{P}_{\mathrm{e}}$ is the mean expected value for a random distribution. The significance of departure from the expected value was tested using the formula $C=r_{o b}-r_{\mathrm{e}} / \sigma \vec{r}_{\mathrm{e}}$, where $C$ is the standard variate for the normal curve. $C$ values of 1.96 and 2.58 represent the 0.05 and 0.01 probability levels of statistical significance for measurements of a population, respectively (Clark and Evans, 1954).

The nearest-neighbor measurements between each artery-innervating cell and its nearest forehead-innervating cell from 2 animals generated $R$ values of 0.20 and 0.47 . The $C$ values for these 2 animals are 10.78 and 5.88 , respectively. These are significant at the $p<0.0002$ level (Clark and Evans, 1954). Similar results were seen when nearest-neighbor measurements were taken between forehead-innervating cells. $R$ values were 0.23 and 0.59 , and $C$ values were 18.63 and 4.4 -both $p<$ 0.0002 . These results demonstrate that the 2 distributions are clumped rather than random. That is, forehead cells are clumped around artery cells, and forehead cells are clumped with respect to each other. However, the distribution of artery cells with respect to one another appears to be random $(R=1.13$ and $1.06, C=1.58$ and 0.69 respectively, both $p>0.05$ ). Figure 2 shows a typical pattern of labeling of artery- and forehead-innervating cells in the trigeminal ganglion. In the case of most ganglion cells that innervate the artery, there are one or more forehead innervating cells nearby.

The ganglion cells innervating the MCA are significantly larger (mean diameter, $28.0 \pm 0.9 \mu \mathrm{m}$ ) than those innervating the forehead (mean diameter, $23.9 \pm 0.8 \mu \mathrm{m} ; t=4.5, d f=218, p<$
0.005). Lawson (1979) has argued that statistically there is evidence for only 2 sizes of primary sensory cell bodies. Figure 3 shows that the population of artery-projecting cells contains a greater proportion of cells in the larger size category than the population of forehead-projecting cells.

\section{Innervation of dura}

Dural nerves have been demonstrated in the monkey (Penfield and McNaughton, 1940) and human (Huber, 1899; Penfield, 1932; Penfield and McNaughton, 1940; Wirth and Van Buren, 1971). However, there has been only one report using axonal transport techniques that demonstrated trigeminal innervation of the dura (Steiger et al., 1982). This report in the cat has been contradicted by the negative findings of othcr investigators using similar techniques in the same species (Mayberg et al., 1981, 1984). In the rat, an extensive innervation of the dura covering the dorsal surface of the brain was seen (Fig. 4). Employing a rostrolateral application on the dorsal dura, the trigeminal labeling was entirely ipsilateral (Fig. $4 D, 2$ rats), with $80-85 \%$ of the cells in the ophthalmic branch. With a more caudomedial application (Fig. 4B,C), cells were retrogradely labeled in the ipsi- and contralateral trigeminal ganglia. The bilateral labeling was most likely due to spreading of TB to the superior dural sinus, as it has been shown to have a bilateral innervation in the cat (Mayberg et al., 1984). TB spread was observed to be greater in these dural applications than in the MCA applications; however, none of the dural applications appeared to spread to any major arteries.

\section{Innervation of the MCA versus dura or MMA}

Fluorescent retrograde double labeling was employed to study the comparative distributions of trigeminal ganglion cells pro- 


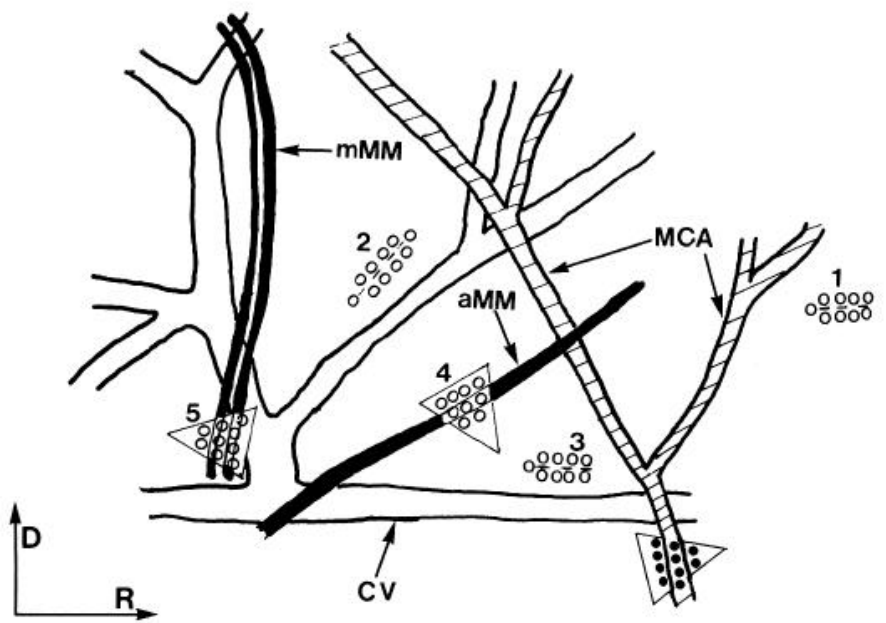

Figure 5. Schematic diagram of intracranial TB and DY application sites. In each application, TB $(\bullet)$ was applied to the MCA and DY (O) to either a dural site or a branch of the MMA $(I-5)$. From this lateral view, branches of the MCA (hatched) and veins (open) are deep in the subarachnoid space, whereas branches of the middle meningeal artery (solid) are more superficial in the dura. Abbreviations: $M C A$, middle cerebral artery; $C V$, cerebral vein; $a M M$, anterior branch of the MMA; $m M M$, middle branch of the MMA; $D$, dorsal; $R$, rostral.

jecting to various intracranial arterial and dural targets (Fig. 5). The MCA applications resulted in a distribution of retrogradely labeled cells similar to that reported above. Within their predominant ophthalmic location in the ganglion, $82 \%$ of the cells innervating the MCA were present in the dorsal half of the ganglion. The MMA representation had a different pattern in the ganglion. The cells were still predominantly in the dorsal half of the ganglia; however, the cells innervating the MMA were seen mostly in the maxillary branch of the ganglion. As reported above, with MCA applications, $60 \pm 5 \%$ of the retrogradely labeled cells were observed in the ophthalmic branch and $38 \pm 5 \%$ in the maxillary branch. In contrast, with MMA applications ( $n=5$ rats), only $24 \pm 11 \%$ of the labeled cells were present in the ophthalmic division and $70 \pm 14 \%$ in the maxillary branch.

In spite of the relative differences in distributions, there was a sizable number of double-labeled cells that projected to both the MCA and either the MMA or various dural sites (Table 1). Double labeling occurred in both the maxillary and ophthalmic branches, with a greater number in the maxillary branch. Figure 6 shows double-labeled cells in the trigeminal ganglion after a TB application to the MCA and a DY application to the aMM. Sites of application were checked by sectioning the brain to make sure there was no overlap of tracer from one application site to another, and only those cases that had no overlap were used. Cells retrogradely labeled with the 2 fluorescent tracers were quite easy to distinguish. The bright yellow nucleus provided an obvious contrast to the blue cytoplasmic label. There were cases in which some DY had leaked out of labeled cells, as nearby glial cells showed some yellow labeling (see Fig. 2). We believe, however, that double labeling was not the result of leakage of tracer from nearby cells for the following reasons. TB was never seen to label glial cells. We have also used TB in longsurvival studies and found that even after $60 \mathrm{~d}$, very little TB leakage occurs (O'Connor and van der Kooy, in press). Furthermore, with dual artery applications TB and DY, labeled cells were usually surrounded by unlabeled cells, and very seldom were cells clumped together. In contrast, in the MCA and forehead experiments, most of the labeled TB and DY cells were clumped near one another, yet less than 1 cell per animal was double labeled.

There was a substantial percentage of double-labeled cells when the 2 arteries received applications. More double-labeled cells were seen when a dural application was close to an artery (i.e., a posterior application site or a site between 2 meningeal artery branches) than when one of the sites was a dural site further away from an artery (Table 1). When double-labeled cells are expressed as a percentage of the total number of cells labeled from the 2 applications, cells from the MCA are much more frequently double labeled when the second tracer is applied to another artery, i.e., MMA (14 $\pm 4 \% ; n=5$ : mean of T145,

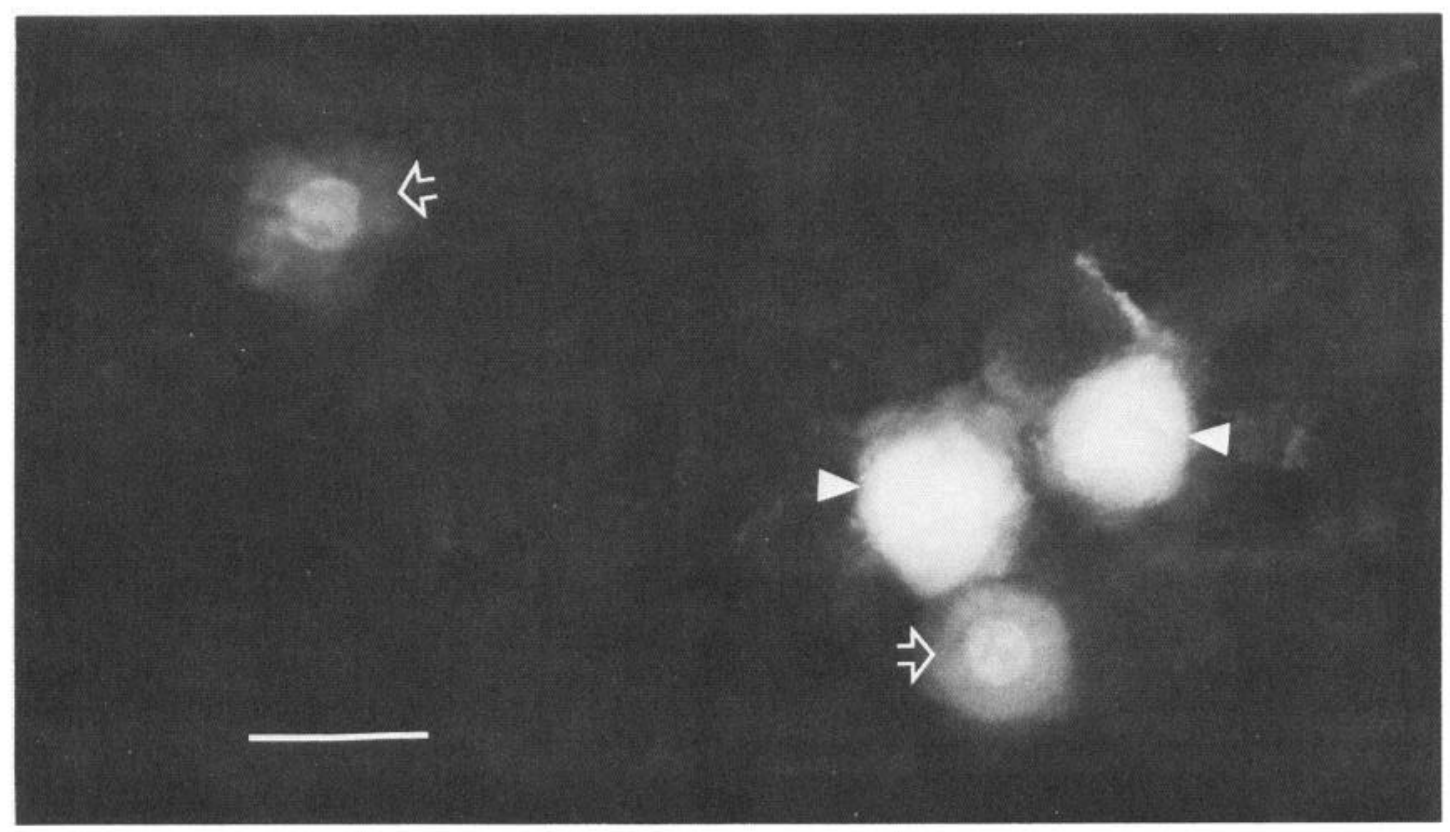

Figure 6. Labeled trigeminal cells after an application of TB to the MCA and DY to the anterior branch of the MMA. Double-labeled cells have both a diffuse TB labeling in their cytoplasm and a bright-yellow nucleus (open arrows). There are also cells that contain only a diffuse TB-stained cytoplasm (arrowheads) or only a bright DY-staining nucleus (not shown). Bar, $30 \mu \mathrm{m}$. 
Table 1. Distribution of labeled cells after concurrent fluorescent tracer application to various arteries and dural sites

\begin{tabular}{|c|c|c|c|c|c|c|c|c|}
\hline \multirow[b]{2}{*}{ Animal } & \multicolumn{5}{|c|}{ Dye application } & \multicolumn{2}{|c|}{ Labeled cells $(n)$} & \multirow{2}{*}{$\begin{array}{l}\text { Percentage } \\
\text { of double- } \\
\text { labeled }\end{array}$} \\
\hline & MCA & $\mathrm{aMM}$ & $\mathrm{mMM}$ & ID & Dura & TB only & DY only & \\
\hline $\mathrm{T} 127$ & $\mathrm{~TB}$ & & & & DY (3) & 28 & 0 & 15.2 \\
\hline T131 & TB & & & & DY(1) & 23 & 10 & 2.9 \\
\hline T136 & TB & & & & DY(1) & 22 & 0 & 0 \\
\hline $\mathrm{T} 145$ & $\mathrm{~TB}$ & DY & & & & 5 & 20 & 3.9 \\
\hline T146 & $\mathrm{TB}$ & DY & & & & 101 & 25 & 8.7 \\
\hline $\mathrm{T} 147$ & TB & DY & & & & 36 & 3 & 26.4 \\
\hline T150 & TB & & & DY & & 39 & 20 & 10.6 \\
\hline $\mathrm{T} 151$ & TB & & & DY & & 28 & 17 & 13.5 \\
\hline $\mathrm{T} 152$ & $\mathrm{~TB}$ & & DY & & & 29 & 40 & 17.9 \\
\hline $\mathrm{T} 153$ & $\mathrm{~TB}$ & & DY & & & 72 & 48 & 11.8 \\
\hline
\end{tabular}

Percentages of double-labeled cells were calculated using the number of double-labeled cells as a percentage of the total number of labeled cells. See Materials and Methods (and Fig. 5) for detailed description of application sites. The numbers in parentheses under Dura refer to application sites schematized in Figure 5.

Abbreviations: MCA, middle cerebral artery; aMM, anterior branch of the middle meningeal artery; mMM, middle branch of the middle meningeal artery; ID, intermediate dura (i.e., the dura between the anterior and middle branches of the middle meningeal artery); TB, True Blue; DY, Diamidimo Yellow.

$146,147,152,153)$ than when the second tracer application is to the dura far removed from an artery (i.e., in front of the MCA, $2 \pm 2 \% ; n=2: \mathrm{T} 131,136)$. There was no difference in the percentage of double-labeled cells after the 2 artery applications ( $14 \pm 4 \%, n=5$ ) compared with the percentage seen after application of TB to the MCA and DY application to the dura between the two meningeal branches, or to the dura just posterior to the MCA $(13 \pm 1 \% ; n=3$ : mean of T127, 150, 151). This shows that it is not a second application site to the dura itself that produces a lack of cells double labeled from an MCA application, but that if the dura application strays too far from a major artery, then the percentage of cells double labeled from the MCA decreases.

When either the aMM or MCA was cauterized proximal to the site of TB application, no TB-labeled cells were seen in the trigeminal ganglion. That the entire effect is lost with a proximal lesion only suggests that no trigeminal fibers run in a distal to proximal direction and that all run in a proximal to distal direction. Therefore, trigeminal nerves do not cross over from the MCA to the aMM and then run proximally to the site of TB application (Fig. 5, no. 4). Also, these results indicate that the ganglion cell labeling is not an artifact of spread of the tracer to the trigeminal ganglion or, alternately, to other trigeminal-innervated targets.

\section{Discussion}

Single trigeminal cells send collaterals to various intracranial arteries and dural sites. Cells innervating the MCA do not send collaterals to the forehead skin, but they are closely associated in the ganglion with cells that innervate the forehead. Based on clinical (Penfield and McNaughton, 1940; Ray and Wolff, 1940; Wirth and Van Buren, 1971) and anatomical (Mayberg et al., 1981; Moskowitz, 1984) data, it has been suggested that the artery innervating cells in the ophthalmic branch of the trigeminal ganglion are involved in referred pain in man. Perhaps the simplest peripheral explanation for referred headache pain is that the same ganglion cells have divergent collateral processes that innervate cerebral arteries and cutaneous receptive fields in the forehead. Our double-labeling experiments show that very few of these cells exist (see also Borges and Moskowitz, 1983). However, there have been 2 anecdotal observations that the artery-innervating cells in the ganglion have a forehead-innervating cell close by (Borges and Moskowitz, 1983; O'Connor and van der Kooy, 1983). In the present report, we have determined that, indeed, the comparative distribution between these 2 populations is not a random one, but more "clumped." That is, artery cells are always accompanied by nearby forehead cells. Furthermore, the distribution of the forehead cells themselves (but not the artery cells by themselves) is also clumped. Development mechanisms are no doubt responsible for the clumping patterns of these different ganglion cells. Perhaps the close association of individual artery-innervating cells with forehead-projecting cells in the ganglion predisposes their central processes to grow together toward the brain stem and establish convergent inputs to second-order trigeminal sensory cells in the brain stem. The activation of these brain-stem cells would then provide the anatomical basis for referred headache pain. Central convergence is one mechanism of referred pain that has been shown to occur in the spinal cord and brain stem $(\mathrm{Hu}$ et al., 1981; Milne et al., 1983). Note that it is not a peripheral mechanism of referred pain (ganglion cells double labeled from the artery and the forehead are very rare), but a central mechanism produced by the peripheral organization of ganglion cells. In this light, it is interesting that in the monkey trigeminal ganglion, functional clusters of cells have been observed. Cells with the same peripheral receptive fields (e.g., forehead) or responding to the same functional stimulus (e.g., nociception) were found grouped together (Lende and Poulos, 1970).

The data suggest that the MCA and forehead are not innervated by collaterals of the same ganglion cells. It could be argued that the failure to observe double-labeled cells was due to a lack of tracer sensitivity or to inconsistency of tracer application. However, the fluorescent retrograde tracers employed have been used by many investigators to reveal double-labeled cells; moreover, we have seen many double-labeled trigeminal ganglion cells with identical tracer applications in neonatal rats (O'Connor and van der Kooy, in press). We have recently shown that these double-labeled cells selectively die during the early postnatal period (O'Connor and van der Kooy, in press). For the most part, the arterial application of tracers must have been consistent, because a relatively constant number of ganglion cells was retrogradely labeled in all the animals with 2 exceptions (T145 and T146 in Table 1).

The central projections of the artery-innervating cells are not known at this time. Using transganglionic transport of HRP from the aorta along the peripheral vagus nerve, Kalia and 
Welles (1980) have shown afferent terminations in visceral brainstem nuclei such as nucleus of the solitary tract and the area postrema. With respect to cerebral arteries, it would be of interest to see if transganglionic transport of wheat germ agglutinin-HRP from the arteries along the trigeminal nerve would also produce afferent terminations in visceral nuclei such as the nucleus of the solitary tract and the area postrema.

The cells innervating the artery tend to be larger (mean diameter, $28 \pm 0.8 \mu \mathrm{m}$ ) than those innervating the forehead (mean diameter, $24 \pm 0.8 \mu \mathrm{m}$ ). This is surprising given that the trigeminal innervation is thought to mediate mainly nociception from the arteries and nociceptive information is thought to be transmitted by smaller ganglion cells. Further, substance P, a putative transmitter for nociception that is found primarily in small cells (Hökfelt, 1975), makes up only a small proportion of the trigeminal innervation of the cerebral arteries (Liu-Chen et al., 1984; O'Connor and van der Kooy, in press). The transmitter type(s) and function(s) of the enriched population of larger trigeminal ganglion cells innervating the cerebral arteries are unknown.

Divergent axonal branching patterns are seen with the individual trigeminal ganglion cells that innervate intracranial arterial and dural targets. A substantial number of cells were found to innervate both the MCA and the MMA. The occurrence of a substantial population of cells projecting to both a subarachnoid (middle cerebral) and a supradural (middle meningeal) artery was surprising. There are at least 2 possible points where the branching of these axons takes place. First, the sensory nerves may travel preferentially along a subarachnoid or supradural artery, but where the arteries cross over one another there may be an exchange of nerves, resulting in an extensive overlap of innervation. Alternatively, the axons may branch more proximally, just after they leave the cell body, and then follow different routes to the different arteries. This latter pathway appears to be the route over which these arteries are innervated. When either the aMM or MCA was cauterized proximal to the tracer application, no labeled ganglion cells were observed. If an exchange of nerves did exist where the arteries cross, some labeled cells would still have been observed after proximal cauterization of one of the arteries. Thus, it appears that the MCA and aMM do not exchange nerves as they cross over one another more distally.

Penfield and McNaughton (1940) concluded that the trigeminal innervation of the middle fossa dura and the middle meningeal vessels was via the nervous meningeus medius and the nervous spinosus (branches of the seccond and third divisions of the trigeminal ganglia). It has been postulated that the innervation of the MCA is via a branch from the ophthalmic division that joins the internal carotid artery at its intracavernous segment, where it lies just medial to the ganglia (Mayberg et al., 1984). We have shown that single cells in the first and second divisions of the ganglia innervate both the MCA and MMA. We suggest that the bifurcating axons of these cells follow the 2 separate routes described by Penfield and McNaughton (1940) and Mayberg et al. (1984).

The results suggest that single trigeminal ganglion cells may not have local intracranial preferences for arterial or dural collateral targets. When the retrograde tracer application was between the 2 meningeal branches or just posterior to the MCA, the absolute numbers of labeled cells were less than when the application was to the MMA (Table 1, cases T127, T150, and T151). However, compared with MMA cells, the percentage of these dural cells that could be double-labeled from the MCA (expressed as a portion of total cells retrogradely labeled) was not different. Thus, although dural innervation is not as dense as arterial innervation, a single trigeminal nerve process running along an artery is just as likely to have a collateral innervating the dura as a collateral innervating another arterial branch.

Very anterior dural applications do not double-label ganglion cells projecting to the MCA. It is possible that this dural innervation arises from collaterals of ganglion cells projecting to other cerebral arteries (for instance, the anterior cerebral artery). However, until this is demonstrated, the existence of trigeminal ganglion cells with exclusively dural projections cannot be unequivocally ruled out.

Innervation of cranial arteries was noted as early as 1898 (Gulland) and clearly demonstrated in papers by Huber (1899) and Penfield (1932). Huber observed 2 classes of nerve bundles: medullated, which he termed sensory, and nonmedullated, which he termed vasomotor. These fibers were described as following the large intracranial arteries in the adventitial layer, and they were also seen to occur in the dura, a distance away from any vessels (Huber, 1899). It was noted that a high degree of branching of these fibers takes place. We have shown that some of these nerves with a divergent branching pattern originate from the trigeminal ganglia, and they have a very wide area of innervation.

The extent of the trigeminal innervation of the dura is more controversial. Mayberg et al. (1984) have shown trigeminal projections to the dural sinus and falx cerebri but not to the lateral dural convexity of the cat (Mayberg et al., 1981, 1984). On the other hand, pain has been produced by stimulation of this lateral dural area in humans (Wirth and Van Buren, 1971), and projections have been shown to the dural floors of the anterior, middle, and posterior fossae and the tentorium cerebelli in the cat (Keller et al., 1985; Steiger et al., 1982). Dorsal root ganglion nerve fibers have also been shown to run along ventral spinal cord pia mater (Dalsgaard et al., 1982; Risling et al., 1984). It has been observed immunohistochemically that these fibers contain substance $P$ and that they innervate the pia by way of the ventral roots (Dalsgaard et al., 1982; Risling et al., 1984). We found that trigeminal ganglion cells project to widespread dural areas in the rat. Dorsal applications resulted in retrogradely labeled cells in all 3 trigeminal ganglion branches, but predominantly in the ophthalmic. As the application approached the midline, some cells were seen in the contralateral ganglion. This is similar to the bilateral superior sagittal sinus innervation reported in the cat (Mayberg et al., 1984). With a more lateral application, the retrogradely labeled cells were found mainly in the ipsilateral ophthalmic division of the ganglion. The failure to find these cells in the cat (Mayberg et al., 1981, 1984) may be due to tracer sensitivity, as we have found (unpublished observations) that TB is more sensitive in mapping dural and arterial projections than the WGA-HRP employed in the cat study.

This collateralized distribution of intracranial sensory innervation from the trigeminal ganglion may partly explain the observation that dural stimulation from widely separated areas produces pain referred to the same area (Wirth and Van Buren, 1971). This type of branched distribution is also consistent with theories of pain from migrainous (Moskowitz, 1984) and cluster headaches (Hardebo, 1984), which emphasize the efferent axon reflexes of branching trigeminal fibers to intracranial vessels (Hardebo, 1984; Moskowitz, 1984) and vasomotor ganglia (Hardebo, 1984).

The development of arterial and dural projections from trigeminal ganglion cells and their involvement in the actual production of vascular disease states (e.g., stroke and headaches) is not known. Some light has been shed on the mechanisms of development (O'Connor and van der Kooy, in press), but the relative roles of intracranial trigeminal fibers in afferent versus efferent processes during headache and stroke remain unresolved.

\section{References}

Borges, L. F., and M. A. Moskowitz (1983) Do intracranial and extracranial trigeminal afferents represent divergent axon collaterals? Neurosci. Lett. 35: 265-270. 
Clark, P. J., and F. C. Evans (1954) Distance to nearest neighbour as a measure of spatial relationships in populations. Ecology 35: 445453.

Clark, P. J., and F. C. Evans (1979) Generalization of a nearest neighbour measure of dispersion for use in $\mathrm{k}$ dimensions. Ecology $60: 316-$ 317.

Dalsgaard, C.-J., M. Risling, and C. Cuello (1982) Immunohistochemical localization of substance $P$ in the lumbosacral spinal pia mater and ventral roots of the cat. Brain Res. 246: 168-171.

Fay, T. (1932) Atypical facial neuralgia, a syndrome of vascular pain. Ann. Otol. 41: 1030-1062.

Gregg, J. M., and A. D. Dixon (1973) Somatotopic organization of the trigeminal ganglion in the rat. Arch. Oral Biol. 18: 487-498.

Gulland, G. L. (1898) The occurrence of nerves on intracranial blood vessels. Br. Med. J. 2: 781-782.

Hardebo, J. E. (1984) The involvement of trigeminal substance $P$ neurons in cluster headache. An hypothesis. Headache 24: 294-304.

Hökfelt, T., J. O. Kellerth, G. Nilsson, and B. Pernow (1975) Substance $P$ : Localization in the central nervous system and in some primary sensory neurons. Science 190: 889-890.

Hu, J. W., J. O. Dostrovsky, and B. J. Sessle (1981) Functional properties of neurons in cat trigeminal subnucleus caudalis (medullary dorsal horn). I. Responses to oral-facial noxious and nonnoxious stimuli and projections to thalamus and subnucleus oralis. J. Neurophysiol. 45: 173-192.

Huber, G. C. (1899) Observations on the innervation of the intracranial vessels. J. Comp. Neurol. 9: 1-25.

Kalia, M., and R. V. Welles (1980) Brainstem projections of the aortic nerve in the cat: A study using tetramethyl benzidine as the substrate for horseradish peroxidase. Brain Res. 188: 23-32.

Keller, J. T., M. C. Saunders, A. Beduk, and J. G. Jollis (1985) Innervation of the posterior fossa dura of the cat. Brain Res. Bull. 14: 97-102.

Lawson, S. N. (1979) The postnatal development of large light and small dark neurons in mouse dorsal root ganglia: A statistical analysis of cell numbers and size. J. Neurocytol. 8: 275-294.

Lea, P. J. (1983) Image analysis system based on a non-dedicated microcomputer. Micron Microscop. Acta 14: 301-306.

Lende, R. A., and D. A. Poulos (1970) Functional localization in the trigeminal ganglion in the monkey. J. Neurosurg. 32: 336-343.

Liu-Chen, L.-Y., S. A. Gillespie, T. V. Norregaard, and M. A. Moskowitz (1984) Co-localization of retrogradely transported wheat germ agglutinin and the putative neurotransmitter substance $P$ within trigeminal ganglion cells projecting to cat middle cerebral artery. $\mathrm{J}$. Comp. Neurol. 225: 187-192.
Mayberg, M., R. S. Langer, N. T. Zervas, and M. A. Moskowitz (1981) Perivascular meningeal projections from cat trigeminal ganglia: Possible pathway for vascular headaches in man. Science 213: 228-230.

Mayberg, M. R., N. T. Zervas, and M. A. Moskowitz (1984) Trigeminal projections to supratentorial pial and dural blood vessels in cats demonstrated by horseradish peroxidase histochemistry. J. Comp. Neurol. 223: 46-56.

Mazza, J. P., and A. D. Dixon (1972) A histological study of chromatolytic cell groups in the trigeminal ganglion of the rat. Arch. Oral Biol. 17: 377-387.

Milne, R. J., R. D. Foremean, G. J. Giesler Jr., and W. D. Willis (1983) Viscerosomatic convergence onto primate spinothalamic neurons: An explanation for referral of pelvic visceral pain. In Advances in Pain Research and Therapy, J. J. Bonica et al., eds., pp. 131-137, Raven, New York.

Moskowitz, M. A. (1984) The neurobiology of vascular head pain. Ann. Neurol. 16: 157-168.

O'Connor, T., and D. van der Kooy (1983) Trigeminal axon collaterals from single cell bodies do not explain referred head pain. Soc. Neurosci. Abstr. 9: 781 .

O'Connor, T., and D. van der Kooy (in press) Cell death organizes the postnatal devclopment of the trigeminal innervation of the cerebral vasculature. Dev. Brain Res.

Penfield, W. (1932) Intracerebral vascular nerves. Arch. Neurol. Psychiatr. $27: 30-44$.

Penfield, W., and McNaughton, F. (1940) Dural headache and innervation of the dura mater. Arch. Neurol. Psychiatr. 44: 43-75.

Pierau, F., G. Fellmer, and D. C. M. Taylor (1984) Somato-visceral convergence in cat dorsal root ganglion neurones demonstrated by double-labelling with fluorescent tracer. Brain Res. 321: 63-70.

Ray, B. S., and H. G. Wolff (1940) Experimental studies on headache. Arch. Surg. 41: 813-856.

Risling, M., C.-J. Dalsgaard, and A. C. Cuello (1984) Invasion of lumbosacral ventral roots and spinal pia mater by substance P-immunoreactive axons after sciatic nerve lesion in kittens. Brain Res. 307: 351-354.

Steiger, H. J., J. M. Tew, Jr., and J. T. Keller (1982) The sensory representation of the dura mater in the trigeminal ganglion of the cat. Neurosci. Lett. 31: 231-236.

Taylor, D. C. M., and F. Pierau (1982) Double fluorescence labeling supports electrophysiological evidence for dichotomizing peripheral sensory nerve fibres in rats. Neurosci. Lett. 33: 1-6.

Wirth, F. P., and J. M. Van Buren (1971) Referral of pain from dural stimulation in man. J. Neurosurg. 34: 630-642. 